\title{
Instabilidade glenoumeral anterior: Revisão sistemática dos desfechos usados no Brasil*
}

\section{Anterior Glenohumeral Instability: Systematic Review of Outcomes Assessment Used in Brazil}

\author{
Jorge Henrique Assunção ${ }^{10}$ Eduardo Angeli Malavolta ${ }^{1}$ Fernando José de Souza ${ }^{1}$ \\ Mauro Emilio Conforto Gracitelli ${ }^{1}$ Arnaldo Amado Ferreira Neto ${ }^{1}$ \\ ${ }^{1}$ Instituto de Ortopedia e Traumatologia, Hospital das Clínicas, \\ Faculdade de Medicina da Universidade de São Paulo (HCFMUSP), \\ São Paulo, SP, Brasil \\ Endereço para correspondência Jorge Henrique Assunção, PhD, \\ Instituto de Ortopedia e Traumatologia, Hospital das Clínicas, Faculdade de \\ Medicina da Universidade de São Paulo (HCFMUSP), São Paulo, \\ SP 05403-000, Brasil (e-mail: drjorgeassuncao@gmail.com).
}

Rev Bras Ortop 2019;54:483-490.

\begin{abstract}
Resumo
Palavras-chave

- ombro

- avaliação de resultado de intervenções terapêuticas

- luxação do ombro/cirurgia

Uma revisão que envolveu as seis principais revistas ortopédicas internacionais foi publicada recentemente. A publicação descreveu as ferramentas usadas para a avaliação dos desfechos no tratamento cirúrgico da luxação anterior recidivante do ombro. Não existe um levantamento que mostre as principais formas de avaliação para essa doença no Brasil. Os autores avaliaram os desfechos usados nos estudos clínicos que envolveram a instabilidade glenoumeral anterior e que foram publicados na última década nos dois principais periódicos ortopédicos brasileiros, Revista Brasileira de Ortopedia e Acta Ortopédica Brasileira. Foi feita uma revisão da literatura na qual foram incluídos todos os artigos clínicos publicados entre 2007 e 2016 que descreveram ao menos uma medida de desfecho antes e após intervenção cirúrgica. Os desfechos avaliados foram amplitude de movimento, força muscular, testes de exame físico, satisfação, retorno ao esporte, exames de imagem, complicações e escalas clínicas. Foram publicados 12 estudos que avaliaram os resultados clínicos do tratamento cirúrgico da instabilidade anterior do ombro. Dez estudos (83\%) eram séries de caso (nível de evidência IV), 1 (8\%) caso-controle (III), e 1 coorte retrospectiva (III). A média de desfechos avaliados por estudo foi de $3,7 \pm 1,7$. A escala Rowe foi usada em 9 estudos (75\%), e 7 publicações (58\%) usaram a escala da University of California Los Angeles (UCLA). Dez estudos (83\%) relataram as complicações relacionadas ao tratamento cirúrgico. A complicação mais frequentemente relatada foi a recidiva da luxação glenoumeral, encontrada em 9 estudos (75\%). Os estudos nacionais usaram preferencialmente escalas consideradas de baixa conficabilidade, responsividade, e consistência interna.
\end{abstract}

\footnotetext{
Trabalho desenvolvido no Grupo de Ombro e Cotovelo, Instituto de Ortopedia e Traumatologia, Hospital das Clínicas, Faculdade de Medicina, Universidade de São Paulo, São Paulo, SP, Brasil. Publicado Originalmente por Elsevier Editora Ltda.
}

recebido

05 de Setembro de 2017 aceito

07 de Dezembro de 2017
DOI https://doi.org/ 10.1016/j.rbo.2017.12.006. ISSN 0102-3616.
Copyright $\odot 2019$ by Sociedade Brasileira License terms de Ortopedia e Traumatologia. Published by Thieme Revinter Publicações Ltda, Rio de Janeiro, Brazil 


\author{
Abstract \\ Keywords \\ - shoulder \\ - evaluation of results \\ of therapeutic \\ interventions \\ - shoulder \\ dislocation/surgery
}

A review involving the six major international orthopedic journals has been published recently. It described the tools used for the evaluation of outcomes in the surgical treatment of recurrent anterior dislocation of the shoulder. There are no studies that exhibit the main outcome tools for this disease in Brazil. The authors evaluated the outcomes of clinical studies involving anterior glenohumeral instability that were published in the last decade in the two leading Brazilian orthopedic journals, Revista Brasileira de Ortopedia e Acta Ortopédica Brasileira. A review of the literature was performed, including all clinical articles published between 2007 and 2016 that described at least one outcome measure before and after some surgical intervention. The outcomes were range of motion, muscle strength, physical examination testing, patient satisfaction, return to sports, imaging, complications, and functional outcomes scores. Twelve studies evaluating the clinical outcomes of surgical treatment for anterior shoulder instability were published. Ten studies (83\%) were case series (level of evidence IV), 1 (8\%) case-control study (III), and 1 retrospective cohort (III). On average, the number of outcomes assessed was $3.7 \pm 1.7$. The Rowe score was used in 9 studies (75\%), and 7 (58\%) used the University of California Los Angeles (UCLA) scale. Ten studies (83\%) reported complications related to surgical treatment. The complication most frequently reported was recurrent instability, found in 9 studies (75\%). The national studies have preferentially used scales considered to be of low reliability, responsiveness, and internal consistency.

\section{Introdução}

O ombro é a articulação que mais comumente sofre luxação, ${ }^{1} \mathrm{e}$ a instabilidade anterior é a forma encontrada mais frequentemente. Apresenta maior incidência em homens jovens, e o seu tratamento é preferencialmente cirúrgico. ${ }^{2-4}$ Encontramos inúmeros estudos na literatura que avaliam os resultados clínicos do tratamento cirúrgico da instabilidade anterior do ombro. A avaliação clínica padronizada é essencial para determinar a eficácia de um tratamento e também para comparar os resultados de diferentes estudos, além de ser fundamental para a pesquisa clínica. ${ }^{5,6}$ Os métodos de avaliação dos resultados do tratamento ortopédico têm sido modificados nos últimos anos. ${ }^{7,8}$ Previamente, a mensuração era feita com base no exame físico, avaliava a mobilidade articular e força muscular. Porém, foram desenvolvidos questionários ou escalas clínicas que aperfeiçoaram a avaliação dos resultados. ${ }^{9,10}$ Entretanto, existe uma grande variação nas ferramentas de mensuração. ${ }^{11}$ São descritas mais de 40 escalas para avaliar a função do ombro ${ }^{12}$ e não existe um consenso quanto ao melhor método para avaliação dos resultados dos pacientes submetidos ao tratamento cirúrgico para instabilidade glenoumeral. ${ }^{13}$

Lukenchuck et al. ${ }^{14}$ publicaram recentemente uma revisão que envolveu as seis principais revistas ortopédicas internacionais e descreveram as ferramentas usadas para a avaliação dos desfechos no tratamento cirúrgico da luxação anterior recidivante do ombro. Não temos um levantamento que mostre as principais formas de avaliação clínica para essa doença no Brasil. Levantamento semelhante já foi feito para as roturas do manguito rotador e demonstrou que escalas consideradas confiáveis, com alta consistência interna e boa responsividade, raramente foram usadas em nosso meio. ${ }^{15}$
Ressaltamos também que a maioria dos instrumentos de avaliação foi desenvolvida e avaliada na língua inglesa. ${ }^{12}$ Para que esses instrumentos sejam usados no Brasil, recomenda-se a tradução, a adaptação cultural, e testes que avaliem as propriedades de medida desses instrumentos, como consistência interna, reprodutibilidade, validade, e responsividade. ${ }^{7} \mathrm{O}$ objetivo desse estudo foi avaliar os desfechos usados nos estudos clínicos sobre o tratamento cirúrgico da instabilidade glenoumeral anterior publicados na última década nos dois principais periódicos ortopédicos brasileiros.

\section{Métodos}

\section{Desenho}

Foi feita uma revisão da literatura nos dois principais periódicos ortopédicos brasileiros, Revista Brasileira de Ortopedia (RBO) e Acta Ortopédica Brasileira. O período abordado foi de uma década (janeiro de 2007-dezembro de 2016). Este estudo foi aprovado pelo Comitê de Ética local sob o número 1258.

\section{Estratégia de Busca}

Inicialmente, a leitura de todos os títulos dos artigos foi feita por um dos autores (J. H. A.), a partir do índice das revistas. Nos casos de dúvida com a leitura do título, o resumo foi avaliado. Dessa maneira, foram excluídos todos os artigos que não envolviam a articulação do ombro e o tratamento da instabilidade glenoumeral. Em seguida, foram lidos os resumos por três autores (J.H. A., E. A. M., e F. J. S.) e, se necessário, o texto completo, para determinar se o artigo se encaixava nos critérios de seleção. Em caso de discordância na seleção de determinado artigo entre os três autores, a inclusão ou não foi definida por consenso (-Fig. 1). 

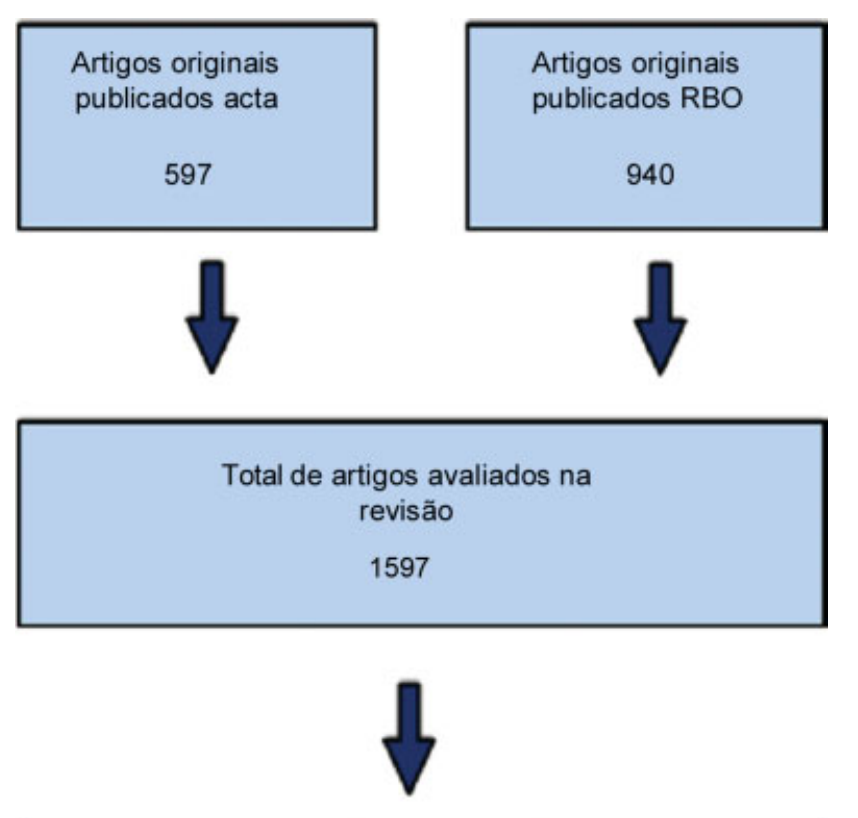

Artigos selecionados pelos títulos

19

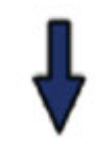

Artigos selecionados após a leitura dos resumos

16

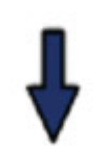

Artigos selecionados após a leitura dos artigos

12

Fig. 1 Algoritmo de pesquisa, 12 artigos foram selecionados baseados nos critérios de inclusão e exclusão.

\section{Critérios de Seleção}

Foram incluídos todos os artigos originais clínicos (ensaio clínico randomizado, coorte, caso-controle e série de casos) que descreveram ao menos uma medida de desfecho após o tratamento cirúrgico para a instabilidade glenoumeral anterior. Não foram incluídos relatos de caso, descrição de técnica cirúrgica, artigos sobre acurácia de métodos diagnósticos, estudos anatômicos, artigos que envolviam animais ou cadáveres, artigos de ciência básica ou de revisão. Foram excluídos artigos que incluíam pacientes com luxação glenoumeral mul- tidirecional ou posterior ou aqueles que avaliavam os resultados do tratamento clínico da instabilidade glenoumeral anterior.

\section{Dados dos Estudos Incluídos}

Foram tabulados os dados referentes ao título do estudo, ano e volume da publicação, casuística, tempo médio de seguimento dos pacientes, seguimento mínimo, seguimento regular e nível de evidência. Em relação ao tratamento cirúrgico, foram coletadas as seguintes informações:

A) Cirurgia por via aberta ou artroscópica;

B) Reparo capsulolabial;

C) Preenchimento do defeito ósseo da glenoide com enxertos ósseos;

D) Se usado enxerto ósseo, qual foi;

E) Tratamento da lesão de Hill-Sachs e a técnica empregada.

\section{Desfechos}

Os resultados clínicos avaliados dos estudos foram os seguintes:

A) Arco de movimento

Foi pesquisado em qual plano ocorreu a avaliação do arco de movimento: flexão frontal, elevação, abdução, rotação lateral (com o braço ao lado do corpo ou abduzido), e rotação medial (com a mão em direção às costas ou com o braço abduzido). A medida em qualquer um dos planos foi tabulada, não foi necessária a avaliação em todos. Os dados só foram considerados descritos se apresentados de maneira quantitativa na seção de resultados. A posição do paciente (supino, sentado ou em posição ortostática) e o uso de goniômetro foram também avaliados.

B) Força muscular

Foi pesquisado em qual plano ocorreu a avaliação da força: flexão frontal, elevação, abdução, rotação lateral (com o braço ao lado do corpo ou abduzido), e rotação medial (com a mão em direção às costas ou com o braço abduzido). A medida em qualquer um dos planos foi tabulada, não foi necessária a avaliação em todos. Os dados deveriam estar apresentados de maneira quantitativa na seção de resultados. A posição do paciente (supino, sentado ou em posição ortostática) e o uso de dinamômetro também foram avaliados. Dados de avaliação manual (graduação de 0-5) também foram computados. Dados referentes a um subdomínio de uma escala clínica foram reportados, se apresentados de maneira individualizada e se fosse possível a extração dos dados referentes à avaliação da força muscular.

C) Testes de exame físico

Foi avaliado o resultado do exame físico dos pacientes, se avaliados e reportados de maneira independente das escalas e questionários de avaliação. Isso incluiu testes de ombro específicos para instabilidade anterior, como o teste da apreensão ou recolocação. A avaliação da força muscular e a amplitude de movimento não foram registradas nessa categoria, mas sim nas categorias acima mencionadas.

D) Satisfação

Qualquer dado referente à satisfação do paciente foi pesquisado. Isso inclui questões referentes à satisfação com o tratamento ou se o paciente recomendaria o procedimento para um terceiro ou se faria novamente a cirurgia. Dados referentes a um subdomínio de uma escala clínica também 
foram reportados se apresentados de maneira individualizada e caso fosse possível a extração dos dados referentes à avaliação da satisfação do paciente.

E) Retorno ao esporte ou ao nível de atividade antes da lesão

Qualquer dado referente ao retorno à atividade esportiva ou nível de atividade prévia à lesão foi pesquisado. Isso incluiu questões objetivas referentes a esse tópico ou o uso de escalas que mensuram esse desfecho.

F) Escalas e questionários de avaliação

Foram avaliados as escalas funcionais e os questionários de avaliação usados pelos autores. Também foram relatados os estudos que usaram a escala visual analógica (EVA) para dor ou função.

\section{G) Complicações}

Todas as complicações relacionadas ao tratamento cirúrgico, como recidiva da instabilidade glenoumeral após o tratamento cirúrgico (apreensão, subluxação ou luxação do ombro), infecção, rigidez, lesão neurovascular, hematomas, osteoartrite, pseudoartrose, e complicações relacionadas aos implantes, foram avaliadas.

H) Imagem

Foram avaliados quais métodos de imagem foram usados (ressonância magnética com ou sem contraste, tomografia computadorizada com ou sem contraste ou radiografias). A periodicidade e temporalidade nas quais foi feito o exame foram reportadas. Descrevemos também a presença ou ausência dos dados relativos à aquisição e análise dos exames de imagem: aparelho usado, avaliadores, imagens obtidas. Para cirurgias que fizeram o preenchimento do defeito ósseo da glenoide com enxertos ósseos, avaliamos a presença de dados referentes ao posicionamento do enxerto e dos parafusos, bem como sinais de consolidação ou pseudoartrose, reabsorção ou fratura do enxerto. Para cirurgias de reparo capsulolabiais, descrevemos os estudos que avaliaram a cicatrização dos reparos.

\section{Análise Estatística}

Os dados foram expostos de maneira descritiva, por meio de número absoluto e percentual, médias e desvio-padrão.

\section{Resultados}

Após serem aplicados os critérios de seleção, foram incluídos 12 estudos que avaliaram os resultados clínicos do tratamento cirúrgico da instabilidade anterior do ombro. Dois (17\%) estudos foram publicados na revista Acta Ortopédica Brasileira, e 10 (83\%) na Revista Brasileira de Ortopedia (- Tabela 1 ). Foram estudados 733 ombros, $61,1 \pm 73,2$ ombros por artigo. $O$ tempo de seguimento médio dos pacientes calculado por média ponderada foi de 35,4 meses. Nenhum estudo fazia seguimento com o tempo de avaliação padronizado, e 5 estudos $^{16-20}(42 \%)$ tinham tempo de seguimento mínimo dos pacientes maior do que 2 anos. Dez estudos ${ }^{17,18,20-27}$ (83\%) eram séries de caso (nível de evidência IV), 1 (8\%) estudo caso-controle $^{16}$ (nível de evidência III), e 1 coorte retrospectivo $^{19}$ (nível de evidência III). Não encontramos estudos randomizados ou coortes prospectivos.

Quatro estudos ${ }^{16,22,24,26}$ (33\%) avaliaram os resultados do tratamento da instabilidade glenoumeral anterior por via aberta, 3 (25\%) estudavam a cirurgia de Latarjet-Patte, ${ }^{16,22,26}$ e $1(8 \%)$ estudou o reparo da lesão labial. ${ }^{24}$ Em 8 publicações, ${ }^{17-21,23,25,27}$ (66\%) o reparo artroscópico das lesões labiais anteriores foi a técnica empregada, um desses avaliava o resultado desse procedimento associado ao remplissage da lesão de Hill-Sachs. ${ }^{23}$ A média de desfechos avaliados por estudo foi de 3,7 \pm 1,7 (-Fig. 2 e - Tabela 2).

\section{Arco de Movimento}

Entre os estudos avaliados, 7 (58\%) relataram a medida do arco do movimento em algum plano no período pós-operatório. ${ }^{16-18,22,23,26,27}$ Os 7 artigos (58\%) apresentavam a rotação lateral, 6 (50\%) avaliaram a rotação medial ${ }^{16-18,22,23,26} \mathrm{e}$ $5,{ }^{18,22,26,27}$ (42\%) estudaram a elevação dos pacientes. Três publicações $^{16,23,27}$ (25\%) avaliavam apenas 2 planos do arco do movimento e $4^{17,18,22,26}$ (33\%) apresentavam as medidas do arco do movimento em 3 planos. A metodologia aplicada na aferição do arco de movimento foi relatada em 3 (25\%) artigos, ${ }^{17,18,26}$ os demais não relataram o uso de goniômetro, técnica da medida ou posição do paciente.

\section{Força}

A força muscular após o tratamento da instabilidade glenoumeral anterior foi aferida em 4 estudos ${ }^{16,20,22,24}$ (33\%). Três (25\%) usaram a mensuração quantitativa da força muscular com auxílio de dinamômetro e relataram a posição do paciente (ortostática ou neutra). Um estudo ${ }^{20}$ (8\%) avaliou apenas a força da elevação dos pacientes, e os demais (25\%) avaliaram a força da rotação medial do ombro. Entre os 3 estudos que avaliaram os resultados da cirurgia de LaterjetPatte, 2 (66\%) avaliaram a força da rotação medial do ombro. $^{16,22}$

\section{Testes de Exame Físico}

Em apenas 4 estudos ${ }^{16,21,22,26}$ (33\%) os pacientes foram avaliados por testes de exame físico posteriormente ao tratamento cirúrgico. Entre as manobras descritas para avaliação da instabilidade glenoumeral anterior, o teste mais frequentemente reportado pelos autores foi o da apreensão, encontrado nos 4 estudos (33\%).

\section{Satisfação e Retorno ao Esporte}

Dos 12 estudos incluídos, nenhum avaliou a satisfação com o tratamento ou se o paciente recomendaria o procedimento para um $3^{\circ}$ ou se faria novamente a cirurgia e apenas $2(17 \%)$ relataram o número de pacientes que retornaram à atividade esportiva prévia, ${ }^{19,26}$ embora nenhum deles tenha usado escalas para mensurar esse desfecho.

\section{Escalas e Questionários de Avaliação}

A escala de Rowe $^{28}$ foi usada em 9 estudos ${ }^{16-19,22-24,26,27}$ (75\%); 7, 19,20,22-24,26 (58\%) usaram a escala da University of California Los Angeles (UCLA), ${ }^{29}$ e $1^{16}$ (8\%) fez a avaliação dos resultados pelo escore de Walch-Duplay(-Fig. 3). Em 7 publicações $^{16,17,19,22-24,26}$ (58\%) foram usadas 2 escalas de avaliação; 3 estudos ${ }^{18,20,27}$ (25\%) apresentaram os resultados por apenas 1 questionário, e $2,{ }^{25}$ (17\%) não usaram escalas ou questionários de avaliação. 
Tabela 1 Publicações sobre o tratamento cirúrgico da instabilidade glenoumeral anterior do ombro entre 2007 e 2016

\begin{tabular}{|c|c|c|c|c|c|}
\hline Autor(es) & Título & Revista & Ano & $\begin{array}{l}\text { Volume } \\
\text { (Número) }\end{array}$ & Páginas \\
\hline $\begin{array}{l}\text { dos Santos } \\
\text { et al. } 16\end{array}$ & $\begin{array}{l}\text { Avaliação da força isométrica e infiltração gordurosa do } \\
\text { subescapular na cirurgia de Latarjet }\end{array}$ & $\begin{array}{l}\text { Acta Ortopédica } \\
\text { Brasileira }\end{array}$ & 2015 & $23(3)$ & $129-133$ \\
\hline $\begin{array}{l}\text { Ferreira } \\
\text { Neto et al. }\end{array}$ & $\begin{array}{l}\text { Tratamento artroscópico da instabilidade anterior do } \\
\text { ombro. Estudo retrospectivo de } 159 \text { casos. }\end{array}$ & $\begin{array}{l}\text { Acta Ortopédica } \\
\text { Brasileira }\end{array}$ & 2011 & $19(1)$ & $41-44$ \\
\hline $\begin{array}{l}\text { Godinho } \\
\text { et al. } 20\end{array}$ & $\begin{array}{l}\text { Avaliação dos resultados funcionais dos ombros submetidos } \\
\text { ao reparo artroscópico de roturas completas do manguito } \\
\text { rotador associadas a luxações traumáticas anteriores }\end{array}$ & $\begin{array}{l}\text { Revista Brasileira } \\
\text { de Ortopedia }\end{array}$ & 2016 & $51(2)$ & $163-168$ \\
\hline $\begin{array}{l}\text { Martel } \\
\text { et al. }\end{array}$ & $\begin{array}{l}\text { Avaliação de resultados pós-operatórios do tratamento } \\
\text { videoartroscópico para luxação recidivante do ombro com } \\
\text { o uso de âncoras metálicas }\end{array}$ & $\begin{array}{l}\text { Revista Brasileira } \\
\text { de Ortopedia }\end{array}$ & 2016 & $51(1)$ & $45-52$ \\
\hline $\begin{array}{l}\text { da Silva } \\
\text { et al. }{ }^{26}\end{array}$ & $\begin{array}{l}\text { Avaliação dos resultados e das complicações em pacientes } \\
\text { com instabilidade anterior de ombro tratados pela técnica } \\
\text { de Latarjet }\end{array}$ & $\begin{array}{l}\text { Revista Brasileira } \\
\text { de Ortopedia }\end{array}$ & 2015 & $50(6)$ & $652-659$ \\
\hline $\begin{array}{l}\text { Godinho } \\
\text { et al. } 19\end{array}$ & $\begin{array}{l}\text { Procedimento artroscópico de Bankart: estudo comparativo } \\
\text { do uso de âncoras com fio duplo ou simples após seguimento } \\
\text { de dois anos }\end{array}$ & $\begin{array}{l}\text { Revista Brasileira } \\
\text { de Ortopedia }\end{array}$ & 2015 & $50(1)$ & $94-99$ \\
\hline $\begin{array}{l}\text { Miyazaki } \\
\text { et al. }{ }^{18}\end{array}$ & $\begin{array}{l}\text { Avaliação dos resultados do tratamento cirúrgico artro- } \\
\text { scópico da instabilidade anterior traumática do ombro com } \\
\text { sutura da lesão na margem cruentizada da cavidade } \\
\text { glenoide }\end{array}$ & $\begin{array}{l}\text { Revista Brasileira } \\
\text { de Ortopedia }\end{array}$ & 2012 & $47(3)$ & $318-324$ \\
\hline $\begin{array}{l}\text { Miyazaki } \\
\text { et al. } 17\end{array}$ & $\begin{array}{l}\text { Avaliação dos resultados do tratamento cirúrgico artroscó- } \\
\text { pico da luxação anterior traumática do ombro: primeiro } \\
\text { episódio }\end{array}$ & $\begin{array}{l}\text { Revista Brasileira } \\
\text { de Ortopedia }\end{array}$ & 2012 & $47(2)$ & $222-227$ \\
\hline $\begin{array}{l}\text { de Almeida } \\
\text { Filho et al. }{ }^{27}\end{array}$ & $\begin{array}{l}\text { Avaliação funcional do reparo artroscópico da instabilidade } \\
\text { anterior recidivante do ombro }\end{array}$ & $\begin{array}{l}\text { Revista Brasileira } \\
\text { de Ortopedia }\end{array}$ & 2012 & $47(2)$ & $214-221$ \\
\hline $\begin{array}{l}\text { Gracitelli } \\
\text { et al. }{ }^{23}\end{array}$ & $\begin{array}{l}\text { Resultados do procedimento artroscópico de remplissage } \\
\text { na luxação anterior recidivante do ombro }\end{array}$ & $\begin{array}{l}\text { Revista Brasileira } \\
\text { de Ortopedia }\end{array}$ & 2011 & $46(6)$ & $684-690$ \\
\hline $\begin{array}{l}\text { Ikemoto } \\
\text { et al. } 22\end{array}$ & $\begin{array}{l}\text { Resultados da cirurgia de Latarjet no tratamento da instabi- } \\
\text { lidade anterior traumática do ombro associada à erosão óssea } \\
\text { da cavidade glenoidal - seguimento mínimo de um ano. }\end{array}$ & $\begin{array}{l}\text { Revista Brasileira } \\
\text { de Ortopedia }\end{array}$ & 2011 & $46(5)$ & $553-560$ \\
\hline $\begin{array}{l}\text { Lech } \\
\text { et al. } 24\end{array}$ & $\begin{array}{l}\text { Integridade do músculo subescapular após a cirurgia } \\
\text { aberta para tratamento da luxac, ão recidivante gle- } \\
\text { noumeral: avaliação clínica e radiológica }\end{array}$ & $\begin{array}{l}\text { Revista Brasileira } \\
\text { de Ortopedia }\end{array}$ & 2009 & $44(5)$ & $420-426$ \\
\hline
\end{tabular}

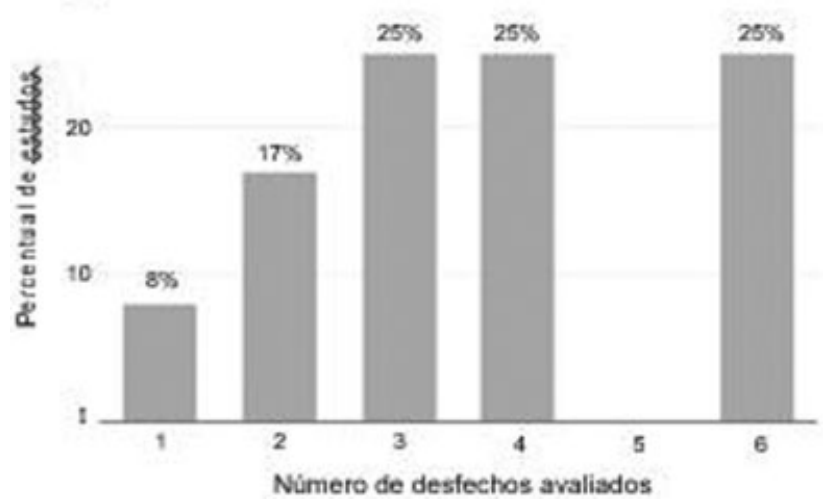

Fig. 2 Distribuição percentual do número de desfechos analisados por estudo.

\section{Complicações}

Dez estudos $^{16-19,21-23,25-27}$ (83\%) relataram as complicações relacionadas ao tratamento cirúrgico. A complicação mais frequentemente relatada foi a recidiva da instabilidade gle-
Tabela 2 Desfechos avaliados e sua frequência

\begin{tabular}{|l|l|}
\hline Desfechos & $\mathbf{n}(\%)$ dos estudos \\
\hline Arco de movimento & $7(58)$ \\
\hline Força & $4(33)$ \\
\hline Exame físico & $4(33)$ \\
\hline Satisfação & $0(0)$ \\
\hline Retorno ao esporte & $2(17)$ \\
\hline Escalas & $10(83)$ \\
\hline Complicacões & $10(83)$ \\
\hline Imagem & $6(50)$ \\
\hline
\end{tabular}

noumeral, encontrada nos 10 estudos (83\%). Sessenta e cinco de 680 pacientes $(9,6 \%)$ permaneceram com os ombros instáveis após o tratamento cirúrgico. Nos pacientes submetidos ao reparo artroscópico das lesões labiais anteriores, 53 de 564 pacientes $(9,3 \%)$ apresentaram recidiva da instabilidade glenoumeral. Por sua vez, 12 de 116 pacientes (10,3\%) 
100

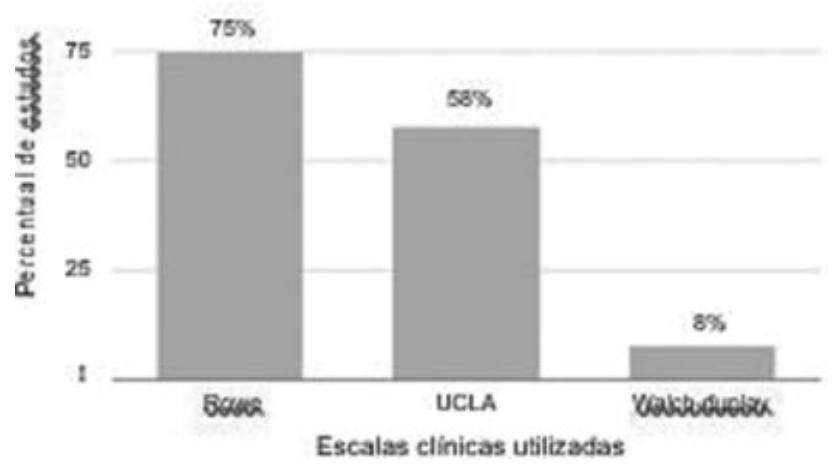

Fig. 3 Percentual de estudos que usaram as escalas clínicas.

submetidos à cirurgia de Latarjet permaneceram com os ombros instáveis. Entre as publicações que relataram essa complicação, apenas $5^{16,21-23,26}$ (42\%) definiram quais pacientes tiveram apreensão, subluxação ou episódios de luxação pós-operatória. Outras complicações reportadas foram dor, capsulite adesiva, âncoras salientes, pseudartrose do processo coracoide, entre outras.

\section{Imagem}

Em 6 estudos ${ }^{16,22-24,26,27}$ (50\%) foram feitos exames de imagem pós-operatórios. 0 exame mais usado foi a radiografia, feita em 3 estudos $22,26,27$ (25\%). Em $2 \operatorname{artigos}^{23,24}$ (16\%) foi usada a ressonância magnética, e em 1 estudo $^{16}$ (8\%) a tomografia computadorizada. Apenas 1 artigo $^{16}(8 \%)$ reportava o aparelho usado, bem como os protocolos de aquisição de imagens. Nenhum estudo informava o número de avaliadores e apenas $2^{16,26}(16 \%)$ relataram a temporalidade da aquisição das imagens. Dois ${ }^{22,26}(66 \%)$ dos 3 artigos que avaliaram cirurgias que fizeram o preenchimento do defeito ósseo da glenoide com enxertos ósseos avaliaram a presença dos dados referentes ao posicionamento do enxerto e dos parafusos, bem como sinais de consolidação ou pseudartrose, reabsorção ou fratura do enxerto. Nenhum estudo avaliou a cicatrização dos reparos capsulolabrais.

\section{Discussão}

A luxação recidivante anterior do ombro corresponde a aproximadamente $8 \%$ dos atendimentos de um cirurgião do ombro e cotovelo ${ }^{28}$ e seu tratamento é preferencialmente cirúrgico. ${ }^{2}$ Entretanto, encontramos apenas 12 estudos que avaliaram os resultados clínicos do tratamento cirúrgico da luxação recidivante anterior do ombro de 2007 a 2016 nos dois principais periódicos ortopédicos brasileiros, uma média de 1,2 estudo por ano. Revistas internacionais de alto fator de impacto, como a Arthroscopy e o American Journal of Sports Medicine, publicaram, nos últimos 5 anos, uma média de 2,4 e 6,6 artigos por ano, respectivamente. ${ }^{14}$

Os estudos incluídos tiveram uma média do tempo de seguimento dos pacientes de 35,4 meses e apenas $42 \%$ dos estudos ${ }^{16-20}$ incluíram casos com tempo de acompanhamento mínimo de dois anos. Esse número é inferior aos estudos internacionais que têm um tempo de seguimento médio de 59,7 meses. ${ }^{14}$ Esse dado é extremamente relevante, pois sabemos que a principal complicação do tratamento da instabilidade glenoumeral anterior é a recidiva e o tempo de acompanhamento é muito importante na avaliação desse desfecho. ${ }^{29}$ Os estudos nacionais também têm um baixo nível de evidência. Não encontramos estudos prospectivos randomizados ou coortes prospectivos e $83 \%$ foram séries de caso (nível de evidência IV). ${ }^{17,18,20-27}$

Cada estudo avaliou em média 3,7 desfechos, número superior ao encontrado em artigos que estudam os resultados do tratamento clínico dos reparos do manguito rotador. ${ }^{15}$ Encontramos também uma grande variabilidade entre os desfechos avaliados pelos diferentes estudos, entretanto a descrição e mensuração das complicações pós-operatórias e escalas ou questionários de avaliação foram empregados em 83\% dos artigos. Esses números são semelhantes aos encontrados nos estudos internacionais, nos quais as complicações e as escalas de avaliação foram usados em $81 \%$ e $88 \%$ dos estudos, respectivamente. $^{14}$

Os desfechos arco de movimento, força, testes de exame físicos e exames de imagem foram menos frequentemente relatados, mas em proporção semelhante aos estudos internacionais. ${ }^{14}$ Entretanto, a técnica empregada, o número de avaliadores e a temporalidade das avaliações comumente não foram descritos. Em relação aos exames de imagem, houve grande variabilidade do exame usado, bem como dos parâmetros usados na avaliação dos desfechos radiográficos, o que pode dificultar o emprego desses dados em revisões sistemáticas. Nenhum estudo relatou a satisfação dos pacientes com o tratamento e apenas duas publicações ${ }^{19,26}$ (17\%) descreveram o número de pacientes que retornaram ao esporte, número inferior aos artigos publicados nos periódicos estrangeiros.

Nos estudos brasileiros, os questionários de avaliação mais usados foram a escala Rowe score for instability e a escala da UCLA, encontrada em 75\% e 58\% dos artigos, respectivamente. Essas ferramentas de avaliação já foram traduzidas e adaptadas culturalmente para língua portuguesa. ${ }^{30,31}$ Entretanto, esses questionários têm diversas ambiguidades em seus itens de avaliação que podem dificultar a resposta do paciente e são consideradas ferramentas não ideais para a pesquisa clínica, devido a inúmeras inconsistências encontradas na sua validade, confiabilidade, e responsividade. ${ }^{13}$

A escala da UCLA foi desenvolvida inicialmente para pacientes submetidos à artroplastia total do ombro. ${ }^{32}$ Ellman et al..$^{33}$ foram os primeiros autores a aplicar essa ferramenta para avaliar pacientes com roturas do manguito rotador; desde então, ela foi usada em várias publicações. Entretanto, não é indicada para avaliar pacientes com luxação glenoumeral, pois não avalia a estabilidade do ombro em seus itens. Nos estudos publicados nas revistas ortopédicas com maior fator impacto, a escala Rowe também é a mais comumente usada, embora seja em uma frequência menor (46\%). O segundo questionário de avaliação mais usado por esses estudos foi o Western Ontario Shoulder Instability (WOSI) index, encontrado em 31\% dos estudos. A escala WOSI é uma ferramenta criada para avaliação exclusivamente de pacientes com instabilidade glenoumeral. ${ }^{34}$ Tem boa responsividade, confiabilidade e validade interna. ${ }^{35}$ 
A escala também é útil para detectar diferenças mínimas significativas entre tratamentos e na evolução clínica dos pacientes. ${ }^{13} \mathrm{O}$ questionário WOSI já foi traduzido e adaptado culturalmente para língua portuguesa, ${ }^{36}$ entretanto, até o momento, não encontramos estudos que avaliem sua validade e confiabilidade na nossa população.

A recidiva da instabilidade é a complicação pós-operatória mais frequentemente reportada, encontrada em dez estudos (83\%). Mas sua definição nos diferentes estudos incluídos bem como na literatura ortopédica ${ }^{37}$ não é muito clara, pode variar desde a apreensão ou subluxação do ombro a episódios de luxação pós-operatória. Como esse parâmetro não é uniforme entre os diferentes artigos, a comparação entre os diferentes estudos é muito difícil e também prejudica a análise desse desfecho em revisões da literatura. Nos estudos nacionais, encontramos que 9,6\% dos ombros permaneceram instáveis após o tratamento cirúrgico. Esse número não difere dos resultados de outras revisões, que relatam a recidiva da instabilidade glenoumeral, de 2 a mais de $20 \%{ }^{29,38}$

Nosso estudo tem algumas limitações. Revisamos os artigos da última década e de apenas dois periódicos nacionais. A nossa estratégia de busca pode ter incluído artigos de autores estrangeiros publicados nos periódicos nacionais e não contemplou artigos de autores brasileiros publicados em periódicos internacionais. Além disso, incluímos todos os tipos de estudos clínicos, portanto nossa revisão tem nível IV de evidência. Entretanto, a maior possibilidade de viés nos artigos de menor nível de evidência não influenciou nossos resultados, uma vez que avaliamos as ferramentas usadas, e não os desfechos em si.

Defendemos que a padronização dos métodos de avaliação dos desfechos no tratamento da instabilidade glenoumeral anterior deve ser estimulada. A avaliação do arco de movimento e da força muscular poderia ser alinhada à usada nos estudos internacionais, bem como o uso de desfechos como a satisfação do paciente com o tratamento e o retorno aos esportes. A escala WOSI, já adaptada culturalmente e traduzida para a língua portuguesa, deveria ser usada pelos estudos nacionais. Esse questionário tem maior responsividade e confiabilidade do que as escalas usadas, Rowe e UCLA. A avaliação dos exames de imagem no período pós-operatório deve ter o método de aquisição, os avaliadores e a temporalidade relatados pelos autores e os métodos de avaliação de imagem para cicatrização dos reparos capsulolabiais ${ }^{39}$ e consolidação dos enxertos ósseos ${ }^{40}$ devem ser padronizados

\section{Conclusão}

Encontramos apenas 12 estudos clínicos publicados entre 2007 a 2016 sobre a instabilidade glenoumeral anterior. 0 número de desfechos avaliados por estudo é 3,7 em média e encontramos uma grande variabilidade entre os desfechos avaliados. As escalas mais empregadas nos estudos foram a escala de Rowe e a escala da UCLA, consideradas de baixa confiabilidade, responsividade, e consistência interna.

\section{Conflitos de Interesse}

Os autores declaram não haver conflitos de interesse.

\section{Referências}

1 Cutts S, Prempeh M, Drew S, Steven D. Anterior shoulder dislocation. Ann R Coll Surg Engl 2009;91(01):2-7

2 Leroux T, Wasserstein D, Veillette C, et al. Epidemiology of primary anterior shoulder dislocation requiring closed reduction in Ontario, Canada. Am J Sports Med 2014;42(02):442-450

3 Owens BD, Dawson L, Burks R, Cameron KL. Incidence of shoulder dislocation in the United States military: demographic considerations from a high-risk population. J Bone Joint Surg Am 2009;91 (04):791-796

4 Handoll HH, Almaiyah MA, Rangan A. Surgical versus non-surgical treatment for acute anterior shoulder dislocation. Cochrane Database Syst Rev 2004;(01):CD004325

5 Gartsman GM, Brinker MR, Khan M. Early effectiveness of arthroscopic repair for full-thickness tears of the rotator cuff: an outcome analysis. JBone Joint Surg Am 1998;80(01):33-40

6 Gartsman GM, Morris BJ, Unger RZ, Laughlin MS, Elkousy HA, Edwards TB. Characteristics of clinical shoulder research over the last decade: a review of shoulder articles in The Journal of Bone \& Joint Surgery from 2004 to 2014. JBone Joint Surg Am 2015;97(05):e26

7 Puga VO, Lopes AD, Costa LO. Avaliação das adaptações transculturais e propriedades de medida de questionários relacionados às disfunções do ombro em língua portuguesa: uma revisão sistemática. Rev Bras Fisioter. 2012;16(02):85-93

8 Brinker MR, Cuomo JS, Popham GJ, O'Connor DP, Barrack RL. An examination of bias in shoulder scoring instruments among healthy collegiate and recreational athletes. JShoulder Elbow Surg 2002;11(05):463-469

9 Garratt A, Schmidt L, Mackintosh A, Fitzpatrick R. Quality of life measurement: bibliographic study of patient assessed health outcome measures. BMJ 2002;324(7351):1417

10 Higginson IJ, Carr AJ. Measuring quality of life: Using quality of life measures in the clinical setting. BMJ 2001;322(7297):1297-1300

11 Makhni EC, Steinhaus ME, Morrow ZS, et al. Outcomes assessment in rotator cuff pathology: what are we measuring? JShoulder Elbow Surg 2015;24(12):2008-2015

12 Harvie P, Pollard TC, Chennagiri RJ, Carr AJ. The use of outcome scores in surgery of the shoulder. JBone Joint Surg Br 2005;87 (02):151-154

13 Kirkley A, Griffin S, Dainty K. Scoring systems for the functional assessment of the shoulder. Arthroscopy 2003;19(10):1109-1120

14 Lukenchuk J, Sims LA, Shin JJ. Variability in outcome reporting for operatively managed anterior glenohumeral instability: a systematic review. Arthroscopy 2017;33(02):477-483

15 Assunção JH, Malavolta EA, Domingues VR, Gracitelli ME, Ferreira Neto AA. Avaliação dos desfechos no tratamento da rotura do manguito rotador: o que usamos no Brasil? Rev Bras Ortop. 2017. Disponível em: http://www.sciencedirect. com/science/article/pii/ S0102361616302764

16 Dos Santos RB, Kauffman FN, de Lima GP, Ferreira AM, Dos Santos SM, Aguiar JL. Evaluation of isometric strength and fatty infiltration of the subscapularis in latarjet surgery. Acta Ortop Bras 2015; 23(03):129-133

17 Miyazaki AN, Fregoneze M, Santos PD, et al. Avaliação dos resultados do tratamento cirúrgico artroscópico da luxação traumática anterior de ombro: primeiro episódio. Rev Bras Ortop 2012;47(02):222-227

18 Miyazaki AN, Fregoneze M, Santos PD, et al. Avaliação dos resultados do tratamento cirúrgico artroscópico da instabilidade anterior traumática do ombro com sutura da lesão na margem cruentizada da cavidade glenoidal. Rev Bras Ortop 2012;47(03):318-324

19 Godinho GG, Freitas JM, França FO, Lago e Santos FM, Aragão AA, Barros MK. Procedimento artroscópico de Bankart: estudo comparativo do uso de âncoras com fio duplo ou simples após seguimento de dois anos. Rev Bras Ortop 2015;50(01):94-99

20 Godinho GG, Freitas JM, de Oliveira França F, Santos FM, de Simoni LF, Godinho PC. Avaliação dos resultados funcionais dos ombros 
submetidos ao reparo artroscópico de roturas completas do manguito rotador associadas a luxações traumáticas anteriores. Rev Bras Ortop 2016;51(02):163-168

21 Ferreira Neto AA, Camanho GL, Felix AM, et al. Tratamento cirúrgico artroscópico da instabilidade anterior do ombro. Estudo Retrospectivo de 159 casos. Acta Ortop Bras 2011;19(01):41-44

22 Ikemoto RY, Murachovisky J, Nascimento LG, et al. Resultados da cirurgia de latarjet no tratamento da instabilidade anterior traumática do ombro associada à erosão óssea da cavidade glenoidal - seguimento mínimo de um ano. Rev Bras Ortop 2011;46(05):553-560

23 Gracitelli ME, Helito CP, Malavolta EA, et al. Resultados do procedimento artroscópico de remplissage na luxação anterior recidivante do ombro. Rev Bras Ortop 2011;46(06):684-690

24 Lech O, Piluski P, Tambani R, Castro N, Pimentel G. Integridade do músculo subescapular após a cirurgia aberta para tratamento da luxação recidivante glenoumeral: avaliação clínica e radiológica. Rev Bras Ortop 2009;44(05):420-426

25 Martel EM, Rodrigues A, Dos Santos Neto FJ, Dahmer C, Ranzzi A, Dubiela RS. Avaliação de resultados pós-operatórios do tratamento videoartroscópico para luxação recidivante de ombro com o uso de âncoras metálicas. Rev Bras Ortop 2016;51(01):45-52

26 da Silva LA, da Costa Lima AG, Kautsky RM, Santos PD, do Val Sella G, Checchia SL. Avaliacão dos resultados e das complicacões em pacientes com instabilidade anterior de ombro tratados pela técnica de Latarjet. Rev Bras Ortop 2015;50(06):652-659

27 de Almeida Filho IA, de Castro Veado MA, Fim M, da Silva Corrêa LV, de Carvalho AE Junior. Avaliação funcional do reparo artroscópico da instabilidade anterior recidivante do ombro. Rev Bras Ortop 2012;47(02):214-221

28 Malavolta EA, Gracitelli MEC, Assunção JH, Pinto GMR, da Silveira AZF, Ferreira AA. Shoulder disorders in an outpatient clinic: an epidemiological study. Acta Ortop Bras 2017;25(03):78-80

29 Gasparini G, De Benedetto M, Cundari A, et al. Predictors of functional outcomes and recurrent shoulder instability after arthroscopic anterior stabilization. Knee Surg Sports Traumatol Arthrosc 2016;24(02):406-413
30 Marcondes FB, de Vasconcelos RA, Marchetto A, de Andrade ALL, Zoppi A, Etchebehere M. Translation and cross-cultural adaptation of the rowe score for portuguese. Acta Ortop Bras 2012;20(06):346-350

31 Oku EC, Andrade AP, Stadiniky SP, Carrera EF, Tellini GG. Tradução e adaptação cultural do Modified-University of California at Los Angeles Shoulder Rating Scale para a língua portuguesa. Rev Bras Reumatol 2006;46(04):246-252

32 Amstutz HC, Sew Hoy AL, Clarke IC. UCLA anatomic total shoulder arthroplasty. Clin Orthop Relat Res 1981;(155):7-20

33 Ellman H, Hanker G, Bayer M. Repair of the rotator cuff. End-result study of factors influencing reconstruction. J Bone Joint Surg Am 1986;68(08):1136-1144

34 Kirkley A, Griffin S, McLintock H, Ng L. The development and evaluation of a disease-specific quality of life measurement tool for shoulder instability. The Western Ontario Shoulder Instability Index (WOSI). Am J Sports Med 1998;26(06):764-772

35 Rouleau DM, Faber K, MacDermid JC. Systematic review of patient-administered shoulder functional scores on instability. J Shoulder Elbow Surg 2010;19(08):1121-1128

36 Barbosa G, Leme L, Saccol MF, Pocchini A, Ejnisman B, Griffin S. Tradução e adaptação cultural para o português do Brasil do Western Ontario Shoulder Instability Index (WOSI). Rev Bras Med Esporte 2012;18(01):35-37

37 Chalmers PN, Mascarenhas R, Leroux T, et al. Do arthroscopic and open stabilization techniques restore equivalent stability to the shoulder in the setting of anterior glenohumeral instability? a systematic review of overlapping meta-analyses. Arthroscopy 2015;31(02):355-363

38 Karataglis D, Agathangelidis F. Long term outcomes of arthroscopic shoulder instability surgery. Open Orthop J 2017;11:133-139

39 Stein T, Mehling AP, Reck C, et al. MRI assessment of the structural labrum integrity after Bankart repair using knotless bio-anchors. Knee Surg Sports Traumatol Arthrosc 2011;19(10):1771-1779

40 Zhu YM, Jiang CY, Lu Y, Li FL, Wu G. Coracoid bone graft resorption after Latarjet procedure is underestimated: a new classification system and a clinical review with computed tomography evaluation. J Shoulder Elbow Surg 2015;24(11):1782-1788 